\title{
Serum levels of ANOS1 serve as a diagnostic biomarker of gastric cancer: a prospective multicenter observational study
}

\author{
Mitsuro Kanda ${ }^{1} \cdot$ Yun-Suhk Suh ${ }^{2} \cdot$ Do Joong Park ${ }^{2} \cdot$ Chie Tanaka $^{1} \cdot$ Sang-Hoon Ahn ${ }^{2} \cdot$ Seong-Ho Kong ${ }^{2} \cdot$ \\ Hyuk-Joon Lee ${ }^{2,3} \cdot$ Daisuke Kobayashi $^{1} \cdot$ Michitaka Fujiwara ${ }^{1} \cdot$ Hideaki Shimada $^{4} \cdot$ BeLong Cho $^{5,6} \cdot$ Kenta Murotani $^{7}$. \\ Hyung-Ho $\mathrm{Kim}^{2} \cdot$ Han-Kwang Yang ${ }^{2,3} \cdot$ Yasuhiro Kodera ${ }^{1}$ (1)
}

Received: 10 May 2019 / Accepted: 26 July 2019 / Published online: 3 August 2019

(c) The International Gastric Cancer Association and The Japanese Gastric Cancer Association 2019

\begin{abstract}
Background Development of high-performance serum biomarkers will likely improve treatment outcomes of patients with gastric cancer (GC). We previously identified the candidate serum markers, anosmin 1 (ANOS1), dihydropyrimidinase-like 3 (DPYSL3), and melanoma-associated antigen D2 (MAGE-D2) and evaluated their clinical significance through a singlecenter retrospective analysis. Here we conducted a prospective multicenter observational study aimed at validating the diagnostic performance of these potential markers.

Methods We analyzed serum levels before and after surgery of the three potential biomarkers in patients with GC and healthy volunteers. Quantification of serum and GC tissue levels was performed using an ELISA.

Results Area under the curve (AUC) values that discriminated patients with GC from healthy controls were $-0.7058,0.6188$, and 0.5031 for ANOS1, DPYSL3, and MAGED2, respectively. The sensitivity and specificity of the ANOS1 assay were 0.36 and 0.85 , respectively. The AUC value of ANOS1 that discriminated patients with stage I GC from healthy controls was 0.7131 . Serum ANOS1 levels were significantly elevated in patients with stage I GC compared with those of healthy controls (median $1179 \mathrm{ng} / \mathrm{ml}$ and $461 \mathrm{ng} / \mathrm{ml}$, respectively, $P<0.0001$ ) and decreased after resection of primary GC lesions $(P<0.0001)$. The combination of serum ANOS1 and DPYSL3 levels increased the AUC value that discriminated patients with GC from healthy controls. Serum levels of ANOS1 did not significantly correlate with those of carcinoembryonic antigen, carbohydrate antigen 19-9, or other markers of inflammation.
\end{abstract}

Conclusions Serum levels of ANOS1 may serve as a useful diagnostic tool for managing GC.

Keywords Gastric cancer · Serum biomarker · Diagnosis · Prospective study

Mitsuro Kanda and Yun-Suhk Suh contributed equally to this work.

Electronic supplementary material The online version of this article (https://doi.org/10.1007/s10120-019-00995-z) contains supplementary material, which is available to authorized users.

Yasuhiro Kodera

ykodera@med.nagoya-u.ac.jp

1 Department of Gastroenterological Surgery (Surgery II), Nagoya University Graduate School of Medicine, 65 Tsurumai-cho, Showa-ku, Nagoya 466-8550, Japan

2 Department of Surgery, Seoul National University College of Medicine, Seoul, Korea

3 Cancer Research Institute, Seoul National University Hospital, Seoul National University College of Medicine, Seoul, Korea

\section{Introduction}

Gastric cancer (GC) remains the third leading cause of cancer death worldwide with a high mortality rate, although its incidence declined during the last decade [1,

4 Department of Gastroenterological Surgery, Graduate School of Medicine, Toho University, Ota City, Japan

5 Department of Family Medicine, Center for Health Promotion and Optimal Aging, Seoul National University College of Medicine and Hospital, Seoul, Korea

6 Institute On Aging, Seoul National University College of Medicine, Seoul, Korea

7 Biostatistics Center, Graduate School of Medicine, Kurume University, Kurume, Japan 
2]. Sensitive biomarkers are necessary for screening, risk classification, and monitoring of patients with GC [3, 4]. Detection through serum tumor markers is a noninvasive, reproducible, and economical diagnostic method that is widely applied in the clinic [5, 6]. However, conventional assays for carcinoembryonic antigen (CEA) and carbohydrate antigen 19-9 (CA19-9) are not sufficiently sensitive or specific for accurate diagnosis of GC, and development of novel biomarkers is therefore warranted [3, 7]. For example, a high-performance biomarker suitable for determining the risk of GC may identify individuals who require further workup. Likewise, a biomarker suitable for predicting metastatic potential may identify a subpopulation of patients most likely to benefit from adjuvant or neoadjuvant treatment [8].

Our previous single-center retrospective study to evaluate biomarkers for GC identified the candidate serum markers for detection or progression of GC as follows: anosmin 1 (ANOS1), dihydropyrimidinase-like 3 (DPYSL3), and melanoma-associated antigen D2 (MAGE-D2) [9-11]. Although we found correlations between the levels of these markers and postoperative prognosis, using optimized cutoff values, their specificities for distinguishing patients with GC patients from healthy individuals, as well as those with other malignancies, were not determined. Moreover, we reasoned that information acquired through longitudinal measurements of serum biomarker levels after a larger multicenter cohort underwent surgery might establish the usefulness of these markers.

Accordingly, we designed and conducted a prospective international multicenter observational study to validate the diagnostic performance of serum levels of ANOS1, DPYSL3, and MAGE-D2 for diagnosis and stratification of disease progression of patients with GC.

\section{Patients and methods}

\section{Study design and registration}

We conducted a prospective multicenter observational study. The scientific and ethical validity of the study protocol was reviewed and approved by the Institutional Review Boards of Nagoya University [IRB No: 2015-0348], Seoul National University Hospital (IRB No: H-1609-056-790), and Seoul National University Bundang Hospital). Written informed consent was obtained from all patients before their enrollment. This study was conducted in accordance with the Declaration of Helsinki (2008) and registered with the University Hospital Medical Information Network (UMIN) Clinical Trial Registry as UMIN000022445 (https://www. umin.ac.jp/ctr/index.htm).

\section{Selection of patients with gastric cancer}

Patients with GC from three institutions were included if they met the eligibility criteria as follows: (1) histologically confirmed primary adenocarcinoma of the stomach (2) age $\geq 20$ years, and (3) no prior treatment. Exclusion criteria included other malignancies and any condition that made the patient unsuitable for inclusion in the study at the discretion of the investigators. Stage classification followed the criteria of the 7th edition of the Union for International Cancer Control (UICC) [12]. Histological subtypes were categorized as follows: differentiated (papillary, well differentiated, and moderately differentiated adenocarcinoma) and undifferentiated (poorly differentiated adenocarcinoma, signet ring cell carcinoma, and mucinous carcinoma).

\section{Healthy controls}

Adults ( $n=66)$ who underwent annual medical examinations (physical examination, blood tests, and chest $\mathrm{X}$-rays) at Nagoya University Hospital and who were not undergoing treatment for any disease were registered as the healthy control group.

\section{Sample collection}

Serum samples $(1 \mathrm{~mL})$ were collected from each patient with GC within 28 days before surgery and 6-12 weeks after surgery. One serum sample was collected from each member of the healthy control group when available. Blood collection was performed using tubes with serum-separating agents, and tubes were maintained at $4{ }^{\circ} \mathrm{C}$ for at least $30 \mathrm{~min}$. The tubes were then centrifuged at $3000 \mathrm{rpm}$ for $5 \mathrm{~min}$, and $1 \mathrm{~mL}$ of serum was added to a sample storage tube. Serum samples were stored at $-80^{\circ} \mathrm{C}$.

\section{ELISA}

Serum levels of ANOS1, DPYSL3, and MAGE-D2 were determined using the ELISA kits as follows: ANOS1 ELISA Kit (CSB-EL011978HU, CUSABIO, Wuhan, China), DPYSL3 ELISA Kit (CSB-EL011790HU, CUSABIO), and MAGE-D2 ELISA Kit (CSB-EL013352HU, CUSABIO), each of which employed a quantitative sandwich enzyme immunoassay technique to detect the human homolog of each protein [9-11]. Samples were tested in duplicate according to the manufacturer's protocol, and the mean values are presented.

\section{Analysis of tissue levels of ANOS1, DPYSL3, and MAGED2}

Primary GC tissues were collected from 43 surgicallyresected specimens, immediately frozen in liquid nitrogen, 
and stored at $-80^{\circ} \mathrm{C}$. Tissues were homogenized and diluted according to the manufacturer's protocol. Serum levels of ANOS1, DPYSL3, and MAGED2 levels were determined using the ELISA kits described above. All tissue samples were tested in duplicate, and the mean values are presented.

\section{External validation}

For external validation of our previous tissue expression data for ANOS1, DPYSL3, and MAGED2 mRNAs, we used an integrated dataset comprising 1065 patients from three major cancer research centers (Berlin, Bethesda, and Melbourne datasets; https://kmplot.com/analysis/) [13].

\section{Statistical analysis}

Qualitative variables were compared between the two patient groups using the $\chi^{2}$ test, and quantitative variables were compared using the Mann-Whitney test. Goodnessof-fit was assessed by calculating the area under the curve (AUC) from receiver operating characteristic (ROC) curve analysis, and the optimal cutoff value was determined using the Youden index. A binary logistic regression was calculated to evaluate predicted probabilities of combinations of two markers. Correlations between the two variables were evaluated using Spearman's rank correlation coefficient. A paired $t$ test was used to compare serum levels of the markers before and after surgery. Statistical analyses were performed using JMP version 13 (SAS Institute, Cary, NC). $P<0.05$ indicates a significant difference.

\section{Results}

\section{Patient selection and backgrounds}

A flow chart of the patient selection procedure is presented in Fig. 1. Between July 2016 and January 2018, 318 patients with GC were screened at Nagoya University, Seoul National University Hospital and Seoul National University Bundang Hospital. Seventeen patients were ineligible. Clinical characteristics of the selected 301 patients with GC are summarized in Table 1. The patient population included 206 men and 95 women (age range, 31-91 years; mean age, $62.8 \pm 12.2$ years). Total gastrectomy was performed to treat 49 (16\%) patients, and 152 and 149 patients were diagnosed with undifferentiated or differentiated GC, respectively. Patients' UICC disease stages were as follows: I, 225 (74\%); II, 47 (16\%); III, 26 (9\%); and IV, 3 (1\%). The latter three patients were assigned this diagnosis according to positive peritoneal lavage cytology.
Screening between July 2016 and January 2018 $\mathrm{n}=318$

- Histologically confirmed adenocarcinoma of the stomach

- Aged $\geq 20$ years

- No other malignancies

- No prior treatment

- Undergoing gastrectomy

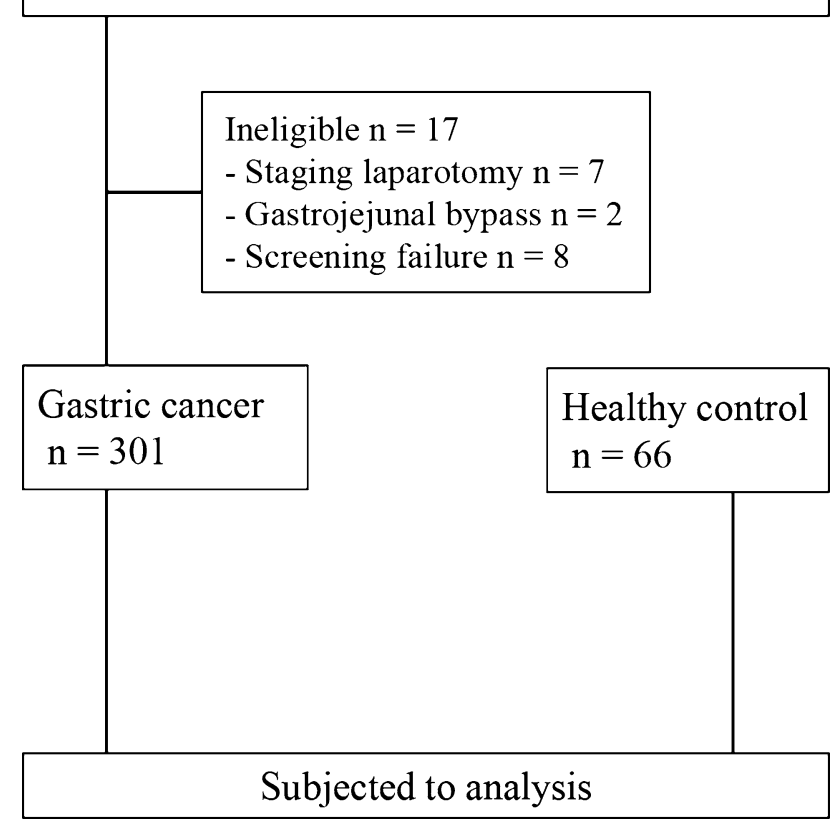

Fig. 1 Study flowchart

\section{Serum levels of ANOS1, DPYSL3, and MAGE-D2 distinguish patients with GC from healthy controls}

ROC curve analysis was performed to evaluate the discriminative ability of each serum marker. The AUC values used to discriminate patients with GC from healthy controls $(n=66)$ for ANOS1, DPYSL3, and MAGED2 were 0.7058 , 0.6188 , and 0.5031 , respectively (Fig. 2a). The sensitivities and specificities were 0.36 and 0.86 for ANOS1, 0.48 and 0.82 for DPYSL3, and 0.28 and 0.92 for MAGED2 (Fig. 2a). Among the 301 patients with GC, the correlation coefficients of serum levels of ANOS1/DPYSL3, DPYSL3/MAGED2, and MAGED2/ANOS1 were 0.4698, 0.2318, and 0.5095, respectively, indicating modest correlations between each pair (Supplemental Fig. 1). When evaluating the capability to discriminate patients with stage I GC $(n=225)$ from healthy controls, the AUC values for ANOS1, DPYSL3, and MAGED2 were $0.7131,0.5948$, and 0.5113 , respectively (Supplementary Fig. 2a). 
Table 1 Characteristics of 301 patients who underwent gastrectomy for gastric cancer

\begin{tabular}{ll}
\hline Variables & Values \\
\hline Age (years), mean \pm standard deviation & $62.8 \pm 12.2$ \\
Sex (male/female) & $206 / 95$ \\
Tumor location & \\
Entire & $4(1 \%)$ \\
Upper third & $59(20 \%)$ \\
Middle third & $88(29 \%)$ \\
Lower third & $150(50 \%)$ \\
Tumor size (mm), mean \pm standard deviation & $32.4 \pm 22.3$ \\
Type of gastrectomy & \\
Total gastrectomy & $49(16 \%)$ \\
Non-total gastrectomy & $252(84 \%)$ \\
Differentiation & \\
Differentiated & $149(49 \%)$ \\
Undifferentiated & $152(51 \%)$ \\
pT factor & \\
pT1 & $217(72 \%)$ \\
pT2 & $32(11 \%)$ \\
pT3 & $24(8 \%)$ \\
pT4 & $27(9 \%)$ \\
pN factor & \\
pN0 & $229(76 \%)$ \\
pN1 & $31(10 \%)$ \\
pN2 & $18(6 \%)$ \\
pN3 & $23(8 \%)$ \\
Iathological stage & \\
II & $225(74 \%)$ \\
IV & $47(16 \%)$ \\
\hline
\end{tabular}

\section{Serum levels of ANOS1, DPYSL3, and MAGE-D2 according to disease stage}

We compared the serum levels of the three markers between the healthy controls and patients with stage I GC or stages II-IV GC. The levels of ANOS1 were significantly elevated in patients with stage I GC compared with those of healthy controls (median $1179 \mathrm{ng} / \mathrm{ml}$ and $461 \mathrm{ng} / \mathrm{ml}$, respectively, $P<0.0001$ ), whereas the levels of patients with stages I and II-V GC were equivalent (Fig. 2b). The levels of DPYSL3 significantly increased in the order of healthy controls (median $142 \mathrm{ng} / \mathrm{ml}$ ), patients with stage I GC (median $230 \mathrm{ng} / \mathrm{ml}$ ), and patients with stage II-IV GC (median $306 \mathrm{ng} / \mathrm{ml}$ ) (Fig. 2b). There were no significant differences between the groups in the levels of MAGED2. Among the 301 patients with GC, there were no significant differences in preoperative serum levels of CEA and CA19-9 between patients with stage I and stages II-IV GC (Supplemental Fig. 2b).

We then focused on the combined serum levels of ANOS1 and DPYSL3. Using the cutoff values determined from ROC curve analysis (ANOS1, $2823 \mathrm{ng} / \mathrm{ml}$ and DPYSL3, $276 \mathrm{ng} /$ $\mathrm{ml}$ ), healthy controls and patients with GC were categorized into the groups as follows: ANOS1 and DPYSL3 doublenegative (< cutoff values); ANOS1 or DPYSL3 positive ( $>$ cutoff values), and ANOS1 and DPYSL3 double-positive. There was no double-positive healthy control (Fig. 2c). The proportion of double-negatives gradually decreased in the order healthy controls, patients with stage I GC, and patients with stages II-IV GC $(80 \%, 43 \%$ and $34 \%$, respectively, $P<0.0001$ ) (Fig. 2c). The AUC values used to discriminate patients with GC from healthy controls for combination of ANOS1 and DPYSL3 were 0.7143 each, slightly, but not significantly higher than that of ANOS1 alone (Supplemental Fig. 2c).

\section{Comparison of preoperative serum levels of ANOS1, DPYSL3, and MAGED2 in patient subgroups}

The serum levels of the three markers among the 301 patients were categorized into subgroups according to clinicopathological parameters. No significant differences between ANOS1 and MAGED2 levels were detected (Table 2). In contrast, pathological vascular invasion and T2-4 tumors were significantly associated with higher levels of preoperative serum DPYSL3 (Table 2).

\section{Correlations with the levels of the indicators of inflammation CEA and CA19-9}

The serum levels of conventional tumor markers (e.g. CEA and CA19-9) are influenced by inflammation. The preoperative serum levels of the three markers did not significantly correlate with white blood cell counts and C-reactive protein levels, indicating that levels of the three markers were independent of inflammation (Supplemental Table 1). Moreover, preoperative levels of the three markers showed no significant correlations with those of CEA and CA19-9 (Supplemental Table 1).

\section{Perioperative changes in serum levels of ANOS1, DPYSL3, and MAGED2}

When we compared the serum levels of ANOS1, DPYSL3, and MAGED2 before and after surgery, we found that they decreased in most patients after resection of the primary GC lesions $(P<0.0001)$ (Fig. 3). 

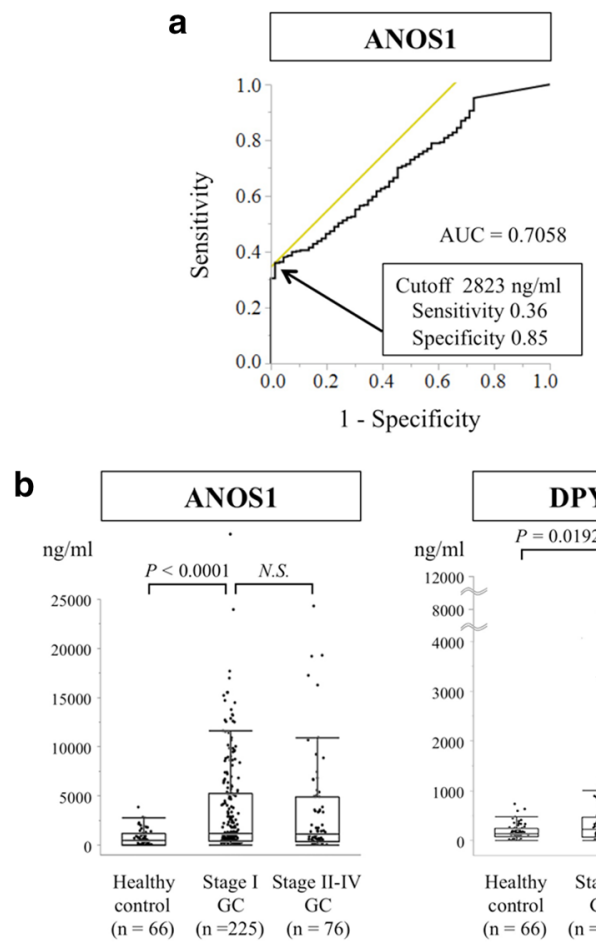
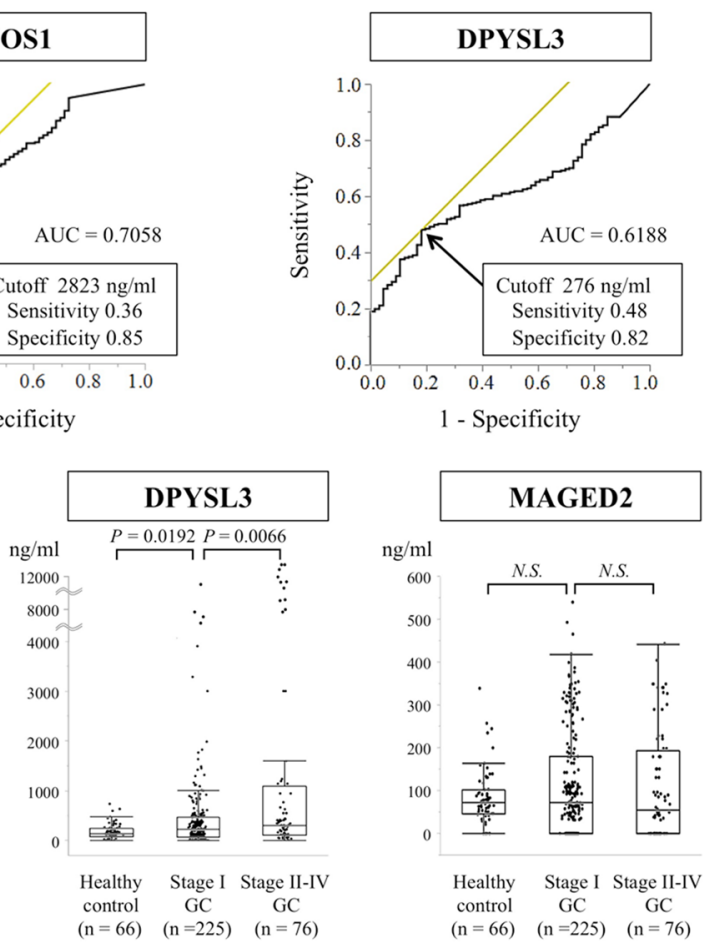

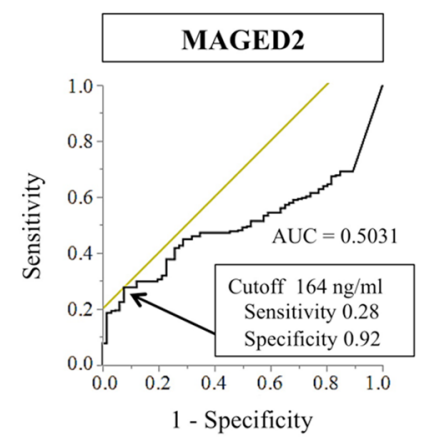

Fig. 2 a Receiver operating characteristic curve analysis of serum levels of the potential markers ANOS1, DPYSL3, and MAGED2 to detect patients with GC. b Serum levels of these markers in healthy

controls and patients with stage I or stages II-IV GC. c Combined serum levels of ANOS1 and DPYSL3 in each group

\section{Levels of ANOS1, DPYSL3, and MAGED2 in primary GC tissues}

Surgically resected primary GC tissues were obtained from 43 (14\%) of the 301 patients with GC. There were no significant correlations between the levels of ANOS1, DPYSL3, and MAGED2 in GC tissues and their respective serum levels (Spearman's correlation coefficients: $-0.0848,0.1690$, and -0.0241 , respectively (Supplemental Fig. 3).

\section{External validation}

We previously found that increased levels of ANOS1, DPYSL3, and MAGED2 mRNAs in primary GC tissues are associated with poor prognosis [9-11]. To confirm the reproducibility of these results, the prognostic significance of tissue levels of the three markers was evaluated using a published database for external validation. This analysis revealed that patients with high levels of ANOS1, DPYSL3, and MAGED2 mRNAs in primary GC tissues experienced significantly shorter overall survival (Supplemental Fig. 4).

\section{Discussion}

Public health programs using barium meals or endoscopy aimed at diagnosis of GC have long been conducted in Japan [14]. Nevertheless, approximately $50 \%$ of patients with GC in Japan present at an advanced stage [15]. One of the reasons for this failure is the limited access to or poor compliance with the program because of the invasive and timeconsuming nature of the examinations. Screening of serum samples is thus an ideal way to select patients for more invasive examinations, provided they achieve sufficient diagnostic performance. Such assays could have further benefit as a method for follow-up and to monitor responses to therapy $[16,17]$. Ideally, such assays will detect elevated levels of serum biomarkers before the onset of disease manifestations and clinical symptoms [18].

Here we conducted a prospective multicenter study to validate the performance of three candidate serum biomarkers that we identified in our previous reports. Among these markers, ANOS1 exhibited the highest AUC value (0.7058) for discriminating patients with GC from healthy controls and achieved diagnostic capacity that was consistent throughout the disease stage, such that Stage I disease could be detected. Further, there were no significant differences between ANOS1 levels between any of the GC subgroups, whereas pathological vascular invasion and T2-4 tumors 
Table 2 Subgroup analyses of preoperative serum levels of ANOS1, DPYSL3 and MAGED2

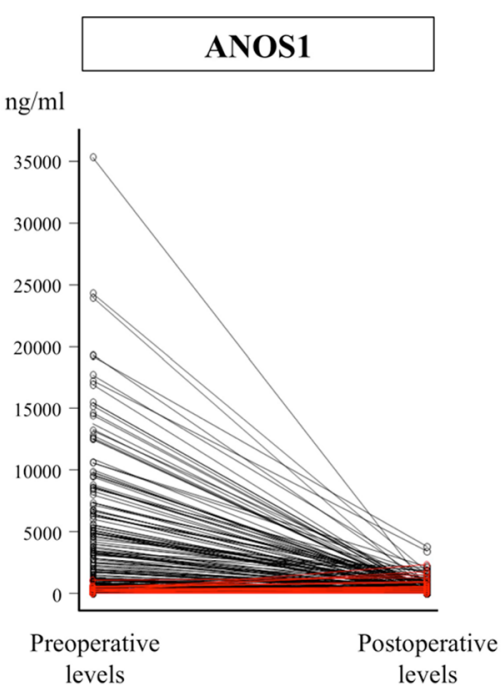

\begin{tabular}{|c|c|c|c|c|c|c|}
\hline & \multicolumn{2}{|l|}{ ANOS1 } & \multicolumn{2}{|l|}{ DPYSL3 } & \multicolumn{2}{|c|}{ MAGED2 } \\
\hline & Median (ng/ml) & $P$ & Median (ng/ml) & $P$ & $\begin{array}{l}\text { Median } \\
(\mathrm{ng} / \mathrm{ml})\end{array}$ & $P$ \\
\hline \multicolumn{7}{|l|}{ Age (year) } \\
\hline$<70$ & 1232 & 0.4590 & 261 & 0.8825 & 68 & 0.5350 \\
\hline$\geq 70$ & 964 & & 238 & & 68 & \\
\hline \multicolumn{7}{|l|}{ Sex (male/female) } \\
\hline Male & 1130 & 0.7627 & 237 & 0.5598 & 71 & 0.1495 \\
\hline Female & 1232 & & 298 & & 66 & \\
\hline \multicolumn{7}{|l|}{ Tumor location } \\
\hline Entire or upper third & 1394 & 0.5151 & 264 & 0.4655 & 70 & 0.7502 \\
\hline Middle or lower third & 1170 & & 252 & & 68 & \\
\hline \multicolumn{7}{|l|}{ Tumor size (mm) } \\
\hline$<30$ & 1271 & 0.1623 & 238 & 0.5344 & 73 & 0.1111 \\
\hline$\geq 30$ & 956 & & 277 & & 60 & \\
\hline \multicolumn{7}{|l|}{ Differentiation } \\
\hline Differentiated & 1179 & 0.7473 & 276 & 0.5862 & 68 & 0.4004 \\
\hline Undifferentiated & 1146 & & 229 & & 70 & \\
\hline \multicolumn{7}{|l|}{ Lymphatic involvement } \\
\hline Absent & 1208 & 0.6731 & 245 & 0.2639 & 72 & 0.4653 \\
\hline Present & 1073 & & 258 & & 61 & \\
\hline \multicolumn{7}{|l|}{ Vascular invasion } \\
\hline Absent & 1170 & 0.7410 & 233 & 0.0225 & 70 & 0.8150 \\
\hline Present & 1187 & & 342 & & 61 & \\
\hline \multicolumn{7}{|l|}{ pT factor } \\
\hline pT1 & 1134 & 0.7351 & 217 & 0.0011 & 72 & 0.4253 \\
\hline pT2-4 & 1362 & & 333 & & 51 & \\
\hline \multicolumn{7}{|l|}{$\mathrm{pN}$ factor } \\
\hline pN0-1 & 1187 & 0.5921 & 255 & 0.5521 & 69 & 0.8435 \\
\hline pN2-3 & 964 & & 223 & & 52 & \\
\hline
\end{tabular}

MAGED2

Media (ng/ml)

\begin{tabular}{|c|c|c|c|c|c|c|}
\hline & \multicolumn{2}{|l|}{ ANOS1 } & \multicolumn{2}{|l|}{ DPYSL3 } & \multicolumn{2}{|c|}{ MAGED2 } \\
\hline & Median (ng/ml) & $P$ & Median (ng/ml) & $P$ & $\begin{array}{l}\text { Median } \\
(\mathrm{ng} / \mathrm{ml})\end{array}$ & $P$ \\
\hline \multicolumn{7}{|l|}{ Age (year) } \\
\hline$<70$ & 1232 & 0.4590 & 261 & 0.8825 & 68 & 0.5350 \\
\hline$\geq 70$ & 964 & & 238 & & 68 & \\
\hline \multicolumn{7}{|l|}{ Sex (male/female) } \\
\hline Male & 1130 & 0.7627 & 237 & 0.5598 & 71 & 0.1495 \\
\hline Female & 1232 & & 298 & & 66 & \\
\hline \multicolumn{7}{|l|}{ Tumor location } \\
\hline Entire or upper third & 1394 & 0.5151 & 264 & 0.4655 & 70 & 0.7502 \\
\hline Middle or lower third & 1170 & & 252 & & 68 & \\
\hline \multicolumn{7}{|l|}{ Tumor size (mm) } \\
\hline$<30$ & 1271 & 0.1623 & 238 & 0.5344 & 73 & 0.1111 \\
\hline$\geq 30$ & 956 & & 277 & & 60 & \\
\hline \multicolumn{7}{|l|}{ Differentiation } \\
\hline Differentiated & 1179 & 0.7473 & 276 & 0.5862 & 68 & 0.4004 \\
\hline Undifferentiated & 1146 & & 229 & & 70 & \\
\hline \multicolumn{7}{|l|}{ Lymphatic involvement } \\
\hline Absent & 1208 & 0.6731 & 245 & 0.2639 & 72 & 0.4653 \\
\hline Present & 1073 & & 258 & & 61 & \\
\hline \multicolumn{7}{|l|}{ Vascular invasion } \\
\hline Absent & 1170 & 0.7410 & 233 & 0.0225 & 70 & 0.8150 \\
\hline Present & 1187 & & 342 & & 61 & \\
\hline \multicolumn{7}{|l|}{ pT factor } \\
\hline pT1 & 1134 & 0.7351 & 217 & 0.0011 & 72 & 0.4253 \\
\hline pT2-4 & 1362 & & 333 & & 51 & \\
\hline \multicolumn{7}{|l|}{$\mathrm{pN}$ factor } \\
\hline pN0-1 & 1187 & 0.5921 & 255 & 0.5521 & 69 & 0.8435 \\
\hline $\mathrm{pN} 2-3$ & 964 & & 223 & & 52 & \\
\hline
\end{tabular}
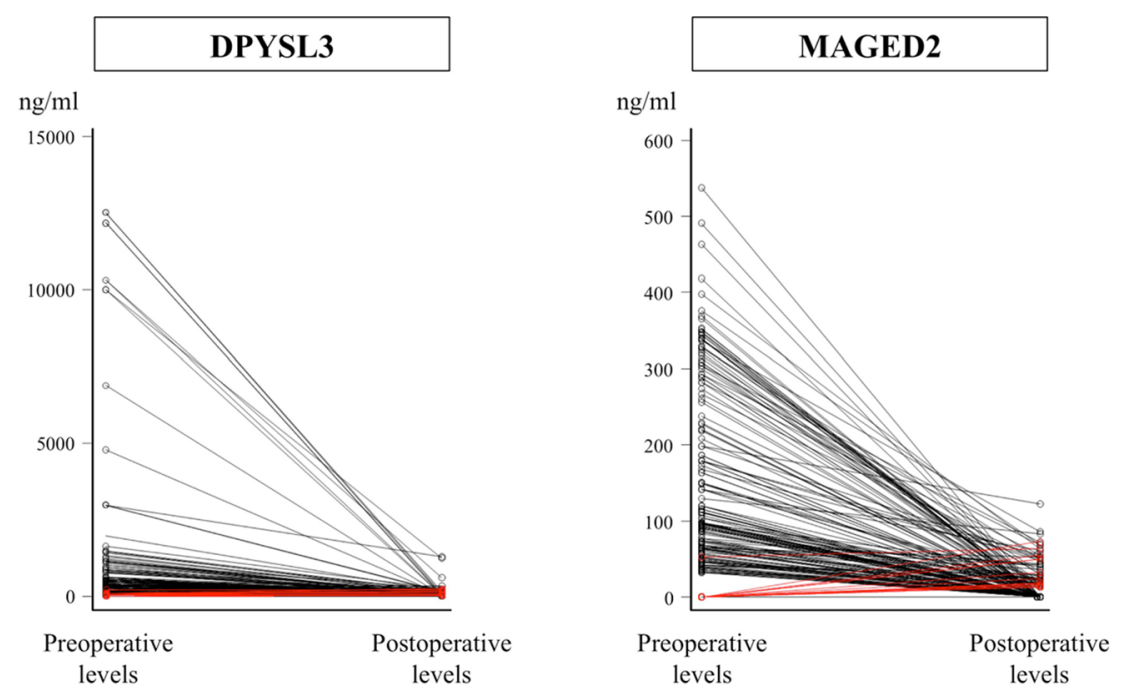

Fig. 3 Perioperative changes in serum levels of ANOS1, DPYSL3, and MAGED2 
were significantly associated with higher levels of preoperative serum DPYSL3. Accordingly, the serum levels of DPYSL3 increased in the order healthy controls, patients with stage I GC, and patients with stages II-IV GC. There were no significant associations between the preoperative levels of the three markers with those of CEA and CA199. Moreover, serum levels of the three markers decreased in most patients after resection of their primary GC lesion. Unfortunately, lack of significant differences between the patient groups excluded MAGED2 levels from further pursuit as a biomarker for GC.

We believe that the ability of serum ANOS1 levels to distinguish between patients with GC of all stages from healthy controls is the most meaningful finding that justifies its further development as a unique biomarker, particularly for diagnosis of early-stage GC. ANOS1 is a secreted 100$\mathrm{kDa}$ glycoprotein that is a component of the extracellular matrix $[19,20]$. In vitro studies show that ANOS1, which binds with high affinity to heparin sulfate proteoglycans in the cell membrane, affects cell adhesion, neurite outgrowth and branching, and cell migration [20, 21]. Similarly, evidence acquired from in vivo studies indicates that ANOS1 regulates neurogenesis as well as the motility and migration of neural cells during development [22, 23]. Further, under physiological and pathological conditions in adults, ANOS1 mediates the outgrowth and genesis of axon collaterals as well as the differentiation of oligodendrocytes and myelin formation [22].

We previously reported that elevated levels of ANOS1 mRNA in tissues are significantly associated with the malignant phenotypes of GC and shorter survival [11]. Further, this single-center retrospective study revealed that ANOS1 levels in serum samples increase in patients with localized GC compared with those of healthy controls [11]. Again, our present prospective multicenter study shows that serum levels of ANOS1 were reproducibly and significantly higher in patients with stage I GC compared with those of healthy controls.

DPYSL3 is a cell-adhesion molecule that is involved in metastasis [24]. Although our ELISA data indicate the potential of DPYSL3 for stratifying patients according to disease progression, its performance as a prognostic determinant could not be fully evaluated, because long-term survival data were unavailable. However, tumor depth, lymph node metastasis, and stage of GC were not significantly associated with serum levels of ANOS1, suggesting that the volume of GC cells did not influence the results, although they may reflect the condition of the gastric mucosa. In contrast, the serum level of DPYSL3 was significantly associated with tumor depth, vascular invasion, and disease stage. When we evaluated the combined levels of ANOS 1 and DPYSL3, the proportion of double-negatives gradually decreased in the order healthy controls, patients with stage I GC, and patients with stages II-IV GC. These findings indicate that this technique is useful for diagnosis and stratification of patients with disease progression.

Emerging evidence indicates the biological roles of the melanoma-associated antigen (MAGE) family in cancer development, progression, and resistance to treatment $[25,26]$. However, little is known about the functions of MAGED2 [27]. In the present study, there were no significant differences in serum levels of MAGED2 between healthy controls and patients with GC. Further, the serum levels of MAGED2 decreased after resection of primary tumors, indicating that MAGED2 was derived from tumors and released into the circulation. However, it will be difficult to use MAGED2 as a serum tumor marker because the changes in its serum levels were small.

Here we show that ANOS1 was the most promising serum biomarker, although the ANOS1 assay was insufficiently sensitive (0.36), even in combination with DPYSL3 (0.39). Markers with high sensitivity with a low false-negative rate, nevertheless, can identify individuals suspected with a disease and are therefore useful for screening. Unfortunately, our data show that ANOS1 is unsuitable for this purpose. In contrast, the ANOS1 assay was highly specific (0.85) and capable of distinguishing healthy controls from patients with GC. Because of a low false-positive rate, an intensive workup to arrive at a definite diagnosis of GC is strongly recommended for ANOS1-positive individuals.

The levels of standard serum tumor markers such as CEA, CA19-9, CA125, and squamous cell carcinoma-related antigen are influenced by inflammation [7, 18, 28]. Serum levels of ANOS1 correlated with white blood cell counts and the levels of C-reactive protein, indicating the absence of a significant association with inflammation. Moreover, serum levels of ANOS1 levels were independent of those of carcinoembryonic antigen and CA19-9, indicating that ANOS1 may serve as a unique screening tool to detect GC. When we analyzed preoperative serum samples of patients with other cancers to determine if the candidate markers were specific to GC, we found that ANOS1 levels were elevated in patients with esophageal or pancreatic cancer, but not in those with colorectal cancer, liver cancer, or breast cancer, indicating that ANOS1 levels may be specific to certain cancers.

To identify the origin of circulating ANOS1, DPYSL3 and MAGED2, we determined their serum levels before and after surgery and found that they were significantly lower after resection of the primary lesions, implicating secretion by tumor cells. However, their serum levels were not significantly associated with those detected in primary GC tissues, indicating that these proteins are not always abundant in serum despite their abundance in gastric cancer tissues. There are possible explanations for this inconsistency. Gastric cancers exhibit high intratumoral heterogeneity [2]. Small pieces of tissue were collected from the edge of the 
tumors and therefore likely did not reflect the levels of certain or all protein in the entire tumor. For example, CEA levels markedly differ between those of gastric cancer tissues and serum $[29,30]$. Thus, the amount of CEA released into the circulation does not mainly depend on tissue CEA levels, but depends on its distribution within a cancerous tissue, tumor differentiation, and lymphovascular invasion $[31,32]$. This may therefore explain discrepancies between serum levels of ANOS1, MAGED2, and DPYSL3 and their respective tissue levels.

A limitation of this study is the use of relatively few healthy controls with demographic backgrounds that did not match those of patients with GC. Further, the prognostic significance of serum levels of ANOS1, DPYSL3, and MAGED2 must be determined, because most patients had stage I GC. Unfortunately, data on long-term survival and recurrence were unavailable during the preparation of this manuscript. Uneven stage distribution of the disease controls may have confounded the comparison of serum levels of the markers across the cancers of the disease control group. Further, we lacked lack of sufficient data to explain the inconsistency between serum and tissue protein levels. Thus, further investigation is required to identify the mechanism that releases these proteins into the circulation. Nevertheless, the reproducibility of determining serum levels of ANOS1 represents an important step towards the development of a novel serum biomarker of GC.

\section{Conclusions}

Measurement of serum levels of ANOS1 may serve as a useful tool for diagnosis of GC. The combination of ANOS1 levels with those of DPYSL3 may enhance the diagnostic performance of the former.

Acknowledgements We thank Edanz Group (www.edanzediting.com/ ac) for editing a draft of this manuscript.

Funding This work was supported by Grants-in-Aid for Scientific Research from the Japanese Society for the Promotion of Science (C, 17K10581), the Funding of the Center for Advanced Medicine and Clinical Research at Nagoya University 2016-2017 and Kurozumi Medical Foundation.

\section{Compliance with ethical standards}

Conflict of interest The authors declare that they have no conflict of interests.

Ethical approval This study conformed to the ethical guidelines of the World Medical Association Declaration of Helsinki, Ethical Principles for Medical Research Involving Human Subjects. Written informed consent for the use of clinical samples and data was obtained from all patients, as required by the Institutional Review Board at Nagoya
University (2014-0043 and 2015-0348), Seoul National University Hospital and Seoul National University Bundang Hospital.

\section{References}

1. Bray F, Ferlay J, Soerjomataram I, Siegel RL, Torre LA, Jemal A. Global cancer statistics 2018: GLOBOCAN estimates of incidence and mortality worldwide for 36 cancers in 185 countries. CA Cancer J Clin. 2018;68:394-424.

2. Van Cutsem E, Sagaert X, Topal B, Haustermans K, Prenen H. Gastric cancer. Lancet. 2016;388:2654-64.

3. Kanda M, Kodera Y. Recent advances in the molecular diagnostics of gastric cancer. World J Gastroenterol. 2015;21:9838-52.

4. Ito M, Oshima Y, Yajima S, Suzuki T, Nanami T, Shiratori F, et al. Is high serum programmed death ligand 1 level a risk factor for poor survival in patients with gastric cancer? Ann Gastroenterol Surg. 2018;2:313-8.

5. Park DJ, Seo AN, Yoon C, Ku GY, Coit DG, Strong VE, et al. Serum VEGF-A and tumor vessel VEGFR-2 levels predict survival in Caucasian but not Asian patients undergoing resection for gastric adenocarcinoma. Ann Surg Oncol. 2015;22(Suppl 3):S1508-S15151515.

6. Kochi R, Yajima S, Nanami T, Suzuki T, Oshima Y, Tokura N, et al. Five-year postsurgical monitoring of serum p53 antibody for locally advanced esophageal squamous cell carcinoma. Clin J Gastroenterol. 2018;11:278-81.

7. He CZ, Zhang KH, Li Q, Liu XH, Hong Y, Lv NH. Combined use of AFP, CEA, CA125 and CA19-9 improves the sensitivity for the diagnosis of gastric cancer. BMC Gastroenterol. 2013;13:87.

8. Leung WK, Wu MS, Kakugawa Y, Kim JJ, Yeoh KG, Goh KL, et al. Screening for gastric cancer in Asia: current evidence and practice. Lancet Oncol. 2008;9:279-87.

9. Kanda M, Nomoto S, Oya H, Shimizu D, Takami H, Hibino S, et al. Dihydropyrimidinase-like 3 facilitates malignant behavior of gastric cancer. J Exp Clin Cancer Res. 2014;33:66.

10. Kanda M, Nomoto S, Oya H, Takami H, Shimizu D, Hibino S, et al. The expression of melanoma-associated antigen D2 both in surgically resected and serum samples serves as clinically relevant biomarker of gastric cancer progression. Ann Surg Oncol. 2016;23(Suppl 2):214-21.

11. Kanda M, Shimizu D, Fujii T, Sueoka S, Tanaka Y, Ezaka K, et al. Function and diagnostic value of Anosmin-1 in gastric cancer progression. Int J Cancer. 2016;138:721-30.

12. Zhang D, Zheng Y, Wang Z, Huang Q, Cao X, Wang F, et al. Comparison of the 7th and proposed 8th editions of the AJCC/UICC TNM staging system for esophageal squamous cell carcinoma underwent radical surgery. Eur J Surg Oncol. 2017;43:1949-55.

13. Szasz AM, Lanczky A, Nagy A, Forster S, Hark K, Green JE, et al. Cross-validation of survival associated biomarkers in gastric cancer using transcriptomic data of 1065 patients. Oncotarget. 2016;7:49322-33.

14. Aikou S, Ohmoto Y, Gunji T, Matsuhashi N, Ohtsu H, Miura $\mathrm{H}$, et al. Tests for serum levels of trefoil factor family proteins can improve gastric cancer screening. Gastroenterology. 2011;141:837-45.e1-7.

15. Katai H, Ishikawa T, Akazawa K, Isobe Y, Miyashiro I, Oda I, et al. Five-year survival analysis of surgically resected gastric cancer cases in Japan: a retrospective analysis of more than 100,000 patients from the nationwide registry of the Japanese Gastric Cancer Association (2001-2007). Gastric Cancer. 2018;21:144-54.

16. Ahn HS, Shin YS, Park PJ, Kang KN, Kim Y, Lee HJ, et al. Serum biomarker panels for the diagnosis of gastric adenocarcinoma. $\mathrm{Br}$ J Cancer. 2012;106:733-9. 
17. Shimada H. p53 molecular approach to diagnosis and treatment of esophageal squamous cell carcinoma. Ann Gastroenterol Surg. 2018;2:266-73.

18. Duraes C, Almeida GM, Seruca R, Oliveira C, Carneiro F. Biomarkers for gastric cancer: prognostic, predictive or targets of therapy? Virchows Arch. 2014;464:367-78.

19. Murcia-Belmonte V, Esteban PF, Garcia-Gonzalez D, De Castro F. Biochemical dissection of Anosmin-1 interaction with FGFR1 and components of the extracellular matrix. J Neurochem. 2010;115:1256-65.

20. Choy CT, Kim H, Lee JY, Williams DM, Palethorpe D, Fellows G, et al. Anosmin-1 contributes to brain tumor malignancy through integrin signal pathways. Endocr Relat Cancer. 2014;21:85-99.

21. de Castro F, Seal R, Maggi R. ANOS1: a unified nomenclature for Kallmann syndrome 1 gene (KAL1) and anosmin-1. Brief Funct Genomics. 2017;16:205-10.

22. Murcia-Belmonte V, Esteban PF, Martinez-Hernandez J, Gruart A, Lujan R, Delgado-Garcia JM, et al. Anosmin-1 overexpression regulates oligodendrocyte precursor cell proliferation, migration and myelin sheath thickness. Brain Struct Funct. 2016;221:1365-85.

23. Qi L, Zhang W, Cheng Z, Tang N, Ding Y. Study on molecular mechanism of ANOS1 promoting development of colorectal cancer. PLoS ONE. 2017;12:e0182964.

24. Rosslenbroich V, Dai L, Baader SL, Noegel AA, Gieselmann V, Kappler J. Collapsin response mediator protein-4 regulates F-actin bundling. Exp Cell Res. 2005;310:434-44.

25. Chomez P, De Backer O, Bertrand M, De Plaen E, Boon T, Lucas S. An overview of the MAGE gene family with the identification of all human members of the family. Cancer Res. 2001;61:5544-51.

26. Xiao J, Chen HS. Biological functions of melanoma-associated antigens. World J Gastroenterol. 2004;10:1849-53.
27. Papageorgio C, Brachmann R, Zeng J, Culverhouse R, Zhang W, McLeod H. MAGED2: a novel p53-dissociator. Int J Oncol. 2007;31:1205-11.

28. Feng F, Tian Y, Xu G, Liu Z, Liu S, Zheng G, et al. Diagnostic and prognostic value of CEA, CA19-9, AFP and CA125 for early gastric cancer. BMC Cancer. 2017;17:737.

29. Uda H, Kanda M, Tanaka C, Kobayashi D, Inaoka K, Tanaka $\mathrm{Y}$, et al. Perioperative serum carcinoembryonic antigen levels predict recurrence and survival of patients with pathological T2-4 gastric cancer treated with curative gastrectomy. Dig Surg. 2018;35:55-63.

30. Yanagawa T, Hayashi Y, Nishida T, Yoshida H, Yura Y, Azuma $\mathrm{M}$, et al. Immunohistochemical demonstration of carcinoembryonic antigen (CEA) on tissue sections from squamous cell head and neck cancer and plasma CEA levels of the patients. Int J Oral Maxillofac Surg. 1986;15:296-306.

31. Maehara Y, Sugimachi K, Akagi M, Kakegawa T, Shimazu H, Tomita M. Serum carcinoembryonic antigen level increases correlate with tumor progression in patients with differentiated gastric carcinoma following noncurative resection. Cancer Res. 1990;50:3952-5.

32. Li M, Li JY, Zhao AL, He JS, Zhou LX, Li YA, et al. Comparison of carcinoembryonic antigen prognostic value in serum and tumour tissue of patients with colorectal cancer. Colorectal Dis. 2009;11:276-81.

Publisher's Note Springer Nature remains neutral with regard to jurisdictional claims in published maps and institutional affiliations. 